High-speed infrared-to-visible up-conversion by free-to-bound transitions in GaP light emitting diodes

This article has been downloaded from IOPscience. Please scroll down to see the full text article.

1985 J. Phys. D: Appl. Phys. 182303

(http://iopscience.iop.org/0022-3727/18/11/020)

View the table of contents for this issue, or go to the journal homepage for more

Download details:

IP Address: 132.199.32.100

The article was downloaded on 25/10/2010 at 11:01

Please note that terms and conditions apply. 


\title{
High-speed infrared-to-visible up-conversion by free-to-bound transitions in GaP light emitting diodes
}

\author{
K Moser, W Eisfeld $\uparrow$, W Penzenstadler and W Prettl \\ Institut für Angewandte Physik, Lniversität Regensburg, D-8400 Regensburg. Federal \\ Republic of Germany
}

Received 19 March 1985, in final form 13 May 1985

\begin{abstract}
In $\mathrm{GaP}: \mathrm{N}(\mathrm{Zn}, \mathrm{Te})$ light emitting diodes electroluminescence and photoluminescence have been excited at $4.2 \mathrm{~K}$ by a $Q$-switched $\mathrm{CO}_{2}$ laser and a pulsed $\mathrm{N}_{2}$ laser. respectively. In the luminescence spectra transitions of free holes to bound donor states could be identified, being responsible for the fast radiative decay on a nanosecond timescale
\end{abstract}

\section{Introduction}

GaP light emitting diodes (LED) can be used as infrared photoconductors and as infraredto-visible up-converters at temperatures below about $40 \mathrm{~K}$ (Eisfeld et al 1983). Carriers excited by infrared radiation from shallow impurities yield a photocurrent and recombine radiatively in the $\mathrm{p}-\mathrm{n}$ junction when the diode is subjected to a forward bias electric field. The spectral distribution of the sensitivity due to continuous wave (CW) infrared illumination has been investigated up to an excitation wavelength of $21 \mu \mathrm{m}$. Under these conditions the structures in the photoconductive spectrum are solely due to the ionisation of shallow donors and the infrared-excited visible emission consists mainly of donoracceptor pair (DAP) luminescence (Moser et al 1985). On the other hand, transient measurements of photoconductivity and up-conversion using pulsed infrared lasers yield fast response times with upper limits of 0.5 and 2 ns, respectively (Moser et al 1984) being too fast for DAP recombination (Vink et al 1974, Pikhtin et al 1970). Moser et al (1984) tentatively attributed the fast radiative recombination to transitions of free holes out of a hot hole distribution into bound donor states which is the only known radiative decay mechanism with a time constant of the order of nanoseconds. In the present paper the decay of infrared-induced electroluminescence and of fluorescence due to pulsed interband excitation has been investigated confirming this interpretation of the fast luminescence component.

\section{Experimental technique}

The experiments were performed on nominally $\mathrm{Zn}$ - and Te-doped GaP:N LED of the same kind as described in Moser et al (1984). Measurements of the infrared photoconductivity spectrum revealed that the diodes contain almost as much $\mathrm{S}$ as the inten-

$\div$ Permanent address: SysScan GmbH, 8025 Unterhaching, Federal Republic of Germany. 
tional n-dopant Te (Moser et al 1985). The samples were immersed in liquid helium and subjected to a forward-bias voltage. A $Q$-switched $\mathrm{CO}_{2}$ laser radiating $200 \mathrm{~ns}$ long pulses with peak intensities of $50 \mathrm{~kW} \mathrm{~cm}^{-2}$ was applied to excite infrared-induced electroluminescence in the diodes. Because no infrared laser emitting shorter radiation pulses was available, the fluorescence was also investigated by interband excitation using a $\mathrm{N}_{2}$ laser which produced $1.7 \mathrm{~ns}$ long pulses in order to improve the time resolution. It was assumed that the decay times of the different luminescence components does not depend on excitation wavelength. The visible radiation of the diodes was spectrally resolved by a monochromator and detected by a Hamamatsu R 928 photomultiplier. The luminescence spectrum was measured during infrared excitation and at a sufficiently long delay time after infrared irradiation by applying a boxcar technique. In addition the spectrum of the photoluminescence power due to interband excitation was determined and the decay of the visible emission was measured for certain fixed wavelengths $\lambda_{\text {det }}$ employing a Biomation transient recorder and averaging over $10^{4}$ excitation pulses.

\section{Experimental results}

\subsection{Luminescence spectra}

In figure $1(a)$ the infrared-induced luminescence spectrum is plotted for $80 \mathrm{~V}$ bias

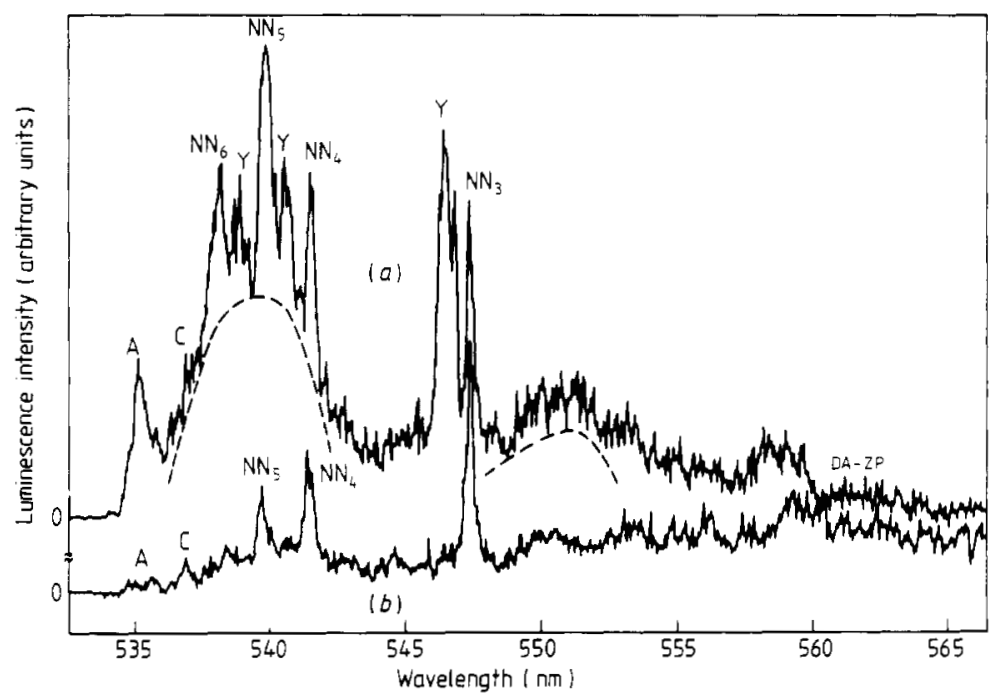

Figure 1. Infrared-induced electroluminescence of $\mathrm{GaP}: \mathrm{N}(\mathrm{Zn}, \mathrm{Te})$ LED with forward bias voltage of $80 \mathrm{~V}$ at $4.2 \mathrm{~K}$, excited by a $Q$-switched $\mathrm{CO}_{2}$ laser. For details see text. $(a)$, Recorded during infrared excitation; gatewidth, $200 \mathrm{~ns}$; no delay. (b), Recorded after infrared excitation gatewidth, $1 \mu \mathrm{s}$; delay, $500 \mathrm{~ns}$.

voltage. The measurement was obtained during infrared irradiation with the $500 \mathrm{~ns}$ gatewidth of the boxcar adjusted to cover the radiation pulse of the $\mathrm{CO}_{2}$ laser. The spectrum shows the well known $\mathrm{A}$ and NN lines which are due to the decay of excitons bound to nitrogen and nitrogen pairs (Cuthbert and Thomas 1967), the Cline, and the 
as yet to be identified, but already observed earlier, lines denoted by Y in figure 1 (Nelson et al 1966, Vink and Peters 1970). All these lines show up on the strong and broad background luminescence indicated in figure $1(a)$ by the broken curves. This background has two broad maxima at about 540 and $550 \mathrm{~nm}$. The structure around $560 \mathrm{~nm}$ results from donor-acceptor pair (DAP) recombination. Figure $1(b)$ shows the corresponding luminescence spectrum measured with a time delay of 500 ns after the infrared pulse and a wide gatewidth of $2 \mu \mathrm{s}$. The A,C and NN lines and the DAP zero phonon line are still present; the broad background luminescence, however, and the $\mathrm{Y}$ lines have disappeared almost totally.

Figure 2 shows the photoluminescence spectrum of an unbiased diode excited by

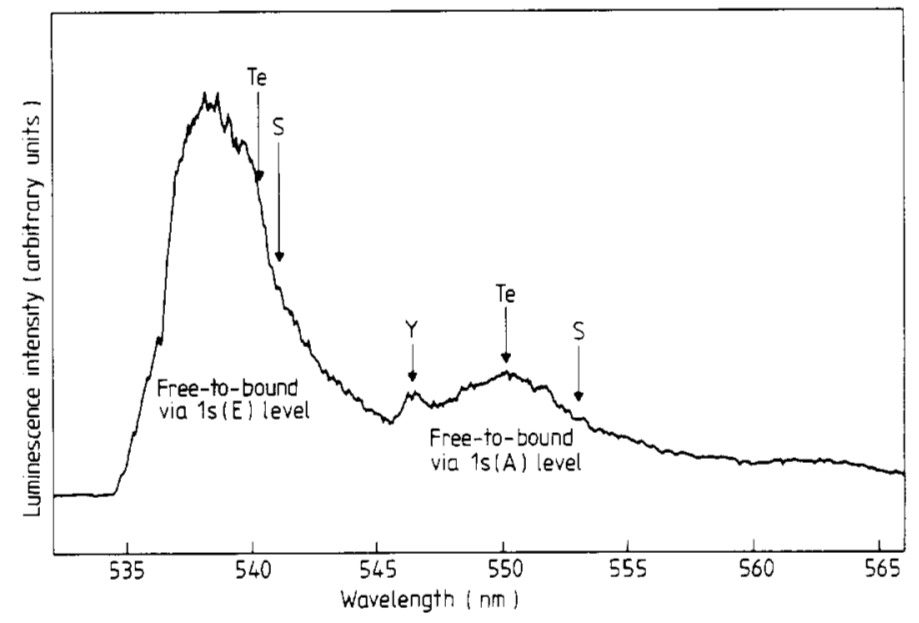

Figure 2. Photoluminescence of $\mathrm{GaP}: \mathrm{N}(\mathrm{Zn}, \mathrm{Te})$ LED excited by a pulsed $\mathrm{N}_{2}$ laser and recorded within a $20 \mathrm{~ns}$ gate without delay. The bias voltage was zero and the temperature $4.2 \mathrm{~K}$. Wavelengths corresponding to the energy separations between the $1 \mathrm{~s}(A)$ and $1 \mathrm{~s}(\mathrm{E})$ Te and $\mathrm{S}$ donor levels and the top of the valence band are indicated by arrows.

pulsed $\mathrm{N}_{2}$ laser radiation. The luminescence was recorded within a gate of $20 \mathrm{~ns}$ without delay relative to the laser pulse. The spectrum shows two distinct broad maxima peaking at 540 and $550 \mathrm{~nm}$ and a weak line at $546.5 \mathrm{~nm}$ without any further fine structure. The broad maxima just correspond to the luminescence background of figure $1(a)$ and the spectral position of the line is in agreement with one of the $Y$ lines. When a non-zero forward-bias voltage was applied to the diode the $\mathrm{C}$ line and the NN lines showed up and the photoluminescence spectrum closely resembled that of the infrared-induced electroluminescence spectrum shown in figure $1(a)$. Measurements with various delay times after interband excitation showed the photoluminescence bands of figure 2 decay with a time constant of less than $10 \mathrm{~ns}$. For delay times longer than $10 \mathrm{~ns}$ and sufficiently large gate width the photoluminescence spectrum consists of the well known donoracceptor recombination structures.

\subsection{Decay times}

The decay of the photoluminescence after interband excitation at zero bias voltage is plotted in figure 3 for detection at different wavelengths. The decay curves in this figure 

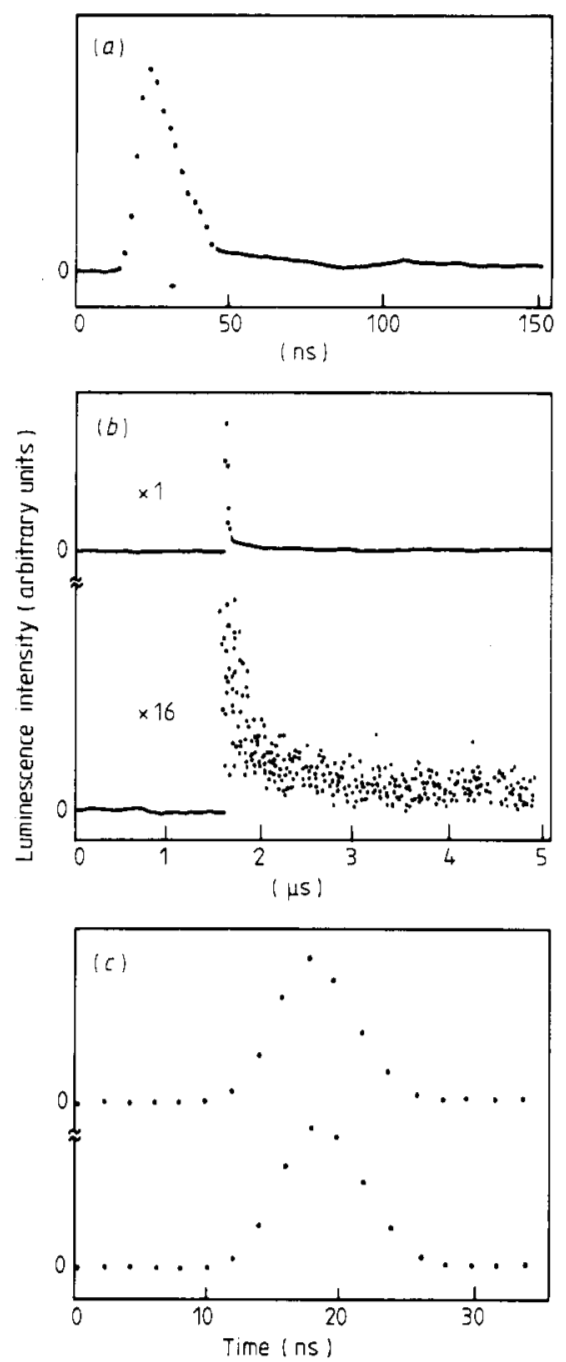

Figure 3. Decay of the photoluminescence of $\mathrm{GaP}: \mathrm{N}(\mathrm{Zn}, \mathrm{Te})$ LED at different detection wavelengths $\lambda_{\text {det }}$. Note the different timescales. (a) $\mathrm{NN}_{4}$ line, $\lambda_{\text {det }}=541.4 \mathrm{~nm}$. (b), Donor-acceptor pair band. Ordinate scale of the lower curve $16 \times$ expanded, $\lambda_{\text {det }}=565 \mathrm{~nm}$. (c), Transitions from free holes to bound donors: upper curve $E_{\mathrm{v}} \rightarrow 1 \mathrm{~s}(\mathrm{~A}), \lambda_{\text {det }}=550 \mathrm{~nm}$; lower curve $E_{\mathrm{v}} \rightarrow 1 \mathrm{~s}(\mathrm{E}), \lambda_{\mathrm{det}}=540 \mathrm{~nm}$.

are selected to show all the typical decay times observed in the present investigation. At the wavelengths of the $\mathrm{NN}$ lines (figure $3(a), \mathrm{NN}_{4}$ ) the decay times vary between 18 and $25 \mathrm{~ns}$ being somewhat shorter than the values given in the literature (Cuthbert and Thomas 1967). Measurements of the $C$ line yield a time constant of $16 \mathrm{~ns}$ which is again smaller than the decay time of $21 \pm 4$ ns observed in the previous investigations (Vink et al 1974, Nelson et al 1966). The DAP fluorescence at $565 \mathrm{~nm}$ (figure $3(b)$ ) shows a strange behaviour, which has not been observed earlier. The well known slow component of fluorescence decaying on a $\mu$ s timescale, which is typical for DAP recombination (Vink et al 1974, Pikhtin et al 1970), is preceded by a very fast decay of the time constant of the order of $10 \mathrm{~ns}$. In figure $3(\mathrm{c})$ the response due to the fluorescence in the centre of the broad bands of figure 2 is shown. In this case the variation of the fluorescence with time could not be resolved. The width of the observed pulses is determined by the band width of the detection device. Thus the decay time of this fluorescence component must be shorter than 4 ns. 


\section{Discussion}

The spectral distribution of the photoluminescence in figure 2 shows a striking similarity to the spectrum of the radiative recombination of an electron-hole plasma in GaP (Shah et al 1977). However the decay time of this process is $30 \mathrm{ns,} \mathrm{much} \mathrm{longer} \mathrm{than} \mathrm{observed}$ in the present case as shown in figure $3(\mathrm{c})$. On the other hand, the rather large concentration of impurities, e.g. $10^{19}$ acceptors per $\mathrm{cm}^{3}$ in the p region of the diode, prevents the formation of electron-hole drops. Thus, recombination of electron-hole drops may be excluded to explain the spectrum in figure 2 . The only conceivable mechanism yielding the observed spectrum and the rapid decay seems to be radiative recombination of free holes and electrons bound to donors (Pikhtin et al 1970, Bindemann et al 1978). The long-wavelength threshold of these free-to-bound transitions is determined by the energy separation of the donor levels and the upper edge of the valence band, $E_{\mathrm{v}}$. These energies have been converted into wavelengths for the $1 \mathrm{~s}(\mathrm{~A})$ and $1 \mathrm{~s}(\mathrm{E})$ donor states of Te and $\mathrm{S}$, the dominant chemical donor species in the present diodes. The spectral positions of these transitions are indicated in figure 2 by arrows, showing that the bands at $540 \mathrm{~nm}$ and at $550 \mathrm{~nm}$ agree well with $E_{\mathrm{v}} \rightarrow E_{1 \mathrm{~s}(\mathrm{E})}$ and $E_{\mathrm{v}} \rightarrow E_{1 \mathrm{~s}(\mathrm{~A})}$, respectively, where $E_{1 \mathrm{~s}(\mathrm{E})}$ and $E_{1 \mathrm{~s}(\mathrm{~A})}$ are the corresponding donor energies (Kopylov and Pikhtin 1978). The maxima of the bands occur at higher quantum energies reflecting a hot-hole distribution in the valence band. It is reasonable to assume a larger capture cross section for the $1 \mathrm{~s}(\mathrm{E})$ donor state because the binding energy of this level is smaller and the wavefunction is more extended than in the case of the $1 \mathrm{~s}(\mathrm{~A})$ state. Thus, as observed experimentally, the band at $540 \mathrm{~nm}$ must be stronger than that at $550 \mathrm{~nm}$. For the $Y$ lines which were present in all the experiments together with the free-to-bound recombination band decaying on the same short time-scale no convincing interpretation could be found.

Finally, the transient behaviour of the DAP fluorescence by $\mathrm{Te}-\mathrm{Zn}$ and $\mathrm{S}-\mathrm{Zn}$ pairs at $565 \mathrm{~nm}$ is to be discussed. As shown in figure 3 we find here a fast component and the characteristic slow decay of the DAP recombination. This can easily be understood because by the free-to-bound mechanism a substantial part of the donor states is rapidly depopulated which also causes a very fast decrease of the rate of donor-acceptor transitions. After relaxation of the hot holes the remaining electrons bound to donors can recombine on the timescale of DAP transitions. This transient behaviour is also a very strong argument for our explanation of the fast luminescence component.

\section{Conclusion}

We have shown that in GaP LED used as infrared-to-visible up-converters slow and fast radiative recombination mechanisms are present leading to a fast and sensitive detection of infrared radiation. Though the long lasting component does not show up on a nanosecond timescale it contributes substantially to the internal quantum efficiency under continuous wave infrared irradiation. The fast luminescence decay could be unambiguously attributed to transitions from free holes to bound donors as anticipated previously (Moser et al 1984). This recombination mechanism could be investigated more thoroughly by infrared pulses in the subnanosecond range because in contrast to conventional photoluminescence measurements the visible emission can easily be discriminated from the exciting infrared radiation. In particular, by this excitation technique the true decay time of the free-to-bound transitions might be resolved. Thus, 
infrared-induced electroluminescence in LED represents a promising method to gain a better understanding of very fast radiative processes in semiconductors.

\section{Acknowledgments}

We thank the Deutsche Forschungsgemeinschaft for financial support. We are also indebted to Dr N Stath and J Attenhauser (Siemens AG) for providing the diode samples.

\section{References}

Bindemann R. Schwabe R and Hänsel T 1978 Phys. Status Solidi b 87169

Cuthbert J D and Thomas D G 1967 Phys. Rev. 154763

Eisfeld W, Werling U and Prettl W 1983 Appl. Phys. Lett. 42276

Kopylov A A and Pikhtin A N 1978 Solid State Commun. 26735

Moser K, Eisfeld W, Werling U, Wahl S and Prettl W 1984 Appl. Phys. Lett. 45711

Moser K, Wahl S, Eisfeld W and Prettl W 1985 J. Appl. Phys. to be published

Nelson D F, Cuthbert J D, Dean P J and Thomas D G 1966 Phys. Rev. Lett. 171262

Pikhtin A N, Yas'kov D A and Glinskii G F 1970 Sov. Phys.-Solid State 12307

Shah J, Leheny R F, Harding W R and Wight D R 1977 Phys. Rev. Lett. 381164

Vink A T, van der Heyden R L A and van Amstel A C 1974 J. Lumin. 9180

Vink A T and Peters R C 1970 J. Lumin. 3209 